\title{
OPEN Persistence of pdm2009-H1N1 internal genes of swine influenza in pigs, Thailand
}

\author{
Chanakarn Nasamran ${ }^{1,2}$, Taveesak Janetanakit ${ }^{1,2}$, Supasama Chiyawong ${ }^{1,2}$, \\ Supanat Boonyapisitsopa ${ }^{1,2}$, Napawan Bunpapong ${ }^{1,2}$, Duangduean Prakairungnamthip ${ }^{1,3}$, \\ Aunyaratana Thontiravong ${ }^{1,3}$ \& Alongkorn Amonsin ${ }^{1,2}$
}

Swine influenza is one of the important zoonotic diseases of pigs. We conducted a longitudinal survey of swine influenza A viruses (S-IAV) circulating in a pig farm with history of endemic S-IAV infection from 2017 to 2018 . The samples were collected from 436 pigs including nasal swab samples $(n=436)$ and blood samples $(n=436)$. Our result showed that $18.81 \%(82 / 436)$ were positive for influenza $A$ virus and subsequently $57 \mathrm{~S}-\mathrm{IAV}$ could be isolated. Then 24 out of $57 \mathrm{~S}-\mathrm{IAVs}$ were selected for whole genome sequencing and could be subtyped as S-IAV-H1N1 $(n=18)$ and S-IAV-H3N2 $(n=6)$. Of 24 S-IAVs, we observed 3 genotypes of S-IAVs including rH1N1 (pdm +1), rH1N1 (pdm + 2), and rH3N2 (pdm + 2). Since all genotypes of S-IAVs in this study contained internal genes from pdmH1N1-2009, it could be speculated that pdmH1N1-2009 was introduced in a pig farm and then multiple reassorted with endemic S-IAVs to generate diversify S-IAV genotypes. Our study supported and added the evidences that pdmH1N1-2009 and it reassortant have predominately persisted in pig population in Thailand. Thus, monitoring of S-IAVs in pigs, farm workers and veterinarians in pig farms is important and should be routinely conducted.

Swine influenza is one of the important zoonotic diseases of pigs. Swine influenza A virus (S-IAV) subtypes $\mathrm{H} 1 \mathrm{~N} 1, \mathrm{H} 3 \mathrm{~N} 2$ and H1N2 cause respiratory disease in pigs worldwide. In Thailand, S-IAV-H1N1 and S-IAV-H3N2 were first reported in the $1970 \mathrm{~s}^{1}$. S-IAV-H1N2 was reported in pig farms in $2005^{2}$. In general, there are 3 major lineages of $\mathrm{H} 1$ gene; Classical swine lineage (CS; $1 \mathrm{~A})$, Eurasian avian-like swine lineage (EA; 1B) and human seasonal (Hu; 1C). The CS (1A) has 6 subclusters; $\alpha$ (1A.1), $\beta$ (1A.2), $\gamma 1$ (1A.3.3.3), $\gamma 2$ (1A.3.2), TR (1A.3.3.1) and pdm09 (1A.3.3.2) ${ }^{3}$. Alpha subclusters (1A.1) could be further classified to subgroups; 1A1.1, 1A.1.2 and 1A.1.3. In Thailand, previous study reported that genotypes of endemic Thai-S-IAV-H1N1 contained H1 from Classical swine lineage (CS) and seven genes from Eurasian avian-like swine lineage (EA) (designated 7+1) or $\mathrm{H} 1$ and NS genes from Classical swine lineage and six genes from Eurasian avian-like swine lineage (designated $6+2)^{4,5}$. For S-IAV-H3N2, there are 2 major lineages of H3 gene; North American lineage and Eurasian swine lineage. The North American lineage can be classified into 4 clades of H3 (clade I, clade II, clade III, clade IV) as well as Human-like swine. The Clade IV of North American S-IAV can be further divided into 6 sub-clusters: A-F. In Thailand, the endemic Thai-S-IAV-H3N2 contained H3 and N2 genes from human-like swine lineage and other internal genes from Eurasian swine lineage (PB1, PB2, PA and M genes) and Classical swine lineage (NP and NS genes) ${ }^{6}$. For S-IAV-H1N2, endemic Thai-S-IAV-H1N2 contains N2 gene from human-like swine lineage, two segments (HA and NS) from Classical swine lineage and other five internal genes (PB2, PB1, PA, $\mathrm{NP}$ and $\mathrm{M}$ ) from Eurasian swine lineage $\mathrm{s}^{5}$.

Genetic diversity of swine influenza viruses has been observed especially in Asian countries. It has been reported that all major S-IAVs lineages from different continents (CS-H1, EA-H1, pdm2009-like H1, American TR, human-like H3, European H3N2, Avian-like viruses etc.) are co-circulating in pigs in Asia including Thailand. Co-circulation of different virus lineages could result in virus reassortment and more genetic diverse of the viruses. After the introduction of pdmH1N1-2009, several studies reported pdmH1N1-2009 infection in pigs $^{7,8}$. Then endemic Thai-S-IAVs were reassorted with pdmH1N1-2009, which contributing triple reassortment internal genes (TRIG). Reassortant S-IAVs with pdmH1N1-2009 became predominant genotypes of S-IAVs in pigs in Thailand and the region ${ }^{9,10}$. Persistence of gene especially pdmH1N1-2009 in pigs for decade

${ }^{1}$ Center of Excellence for Emerging and Re-emerging Infectious Diseases in Animals, Faculty of Veterinary Science, Chulalongkorn University, Bangkok, Thailand. ${ }^{2}$ Department of Veterinary Public Health, Faculty of Veterinary Science, Chulalongkorn University, Bangkok 10330, Thailand. ${ }^{3}$ Department of Microbiology, Faculty of Veterinary Science, Chulalongkorn University, Bangkok, Thailand. ${ }^{\circledR}$ email: Alongkorn.a@chula.ac.th 
could contribute the generation of a novel virus with high infectivity and more virulence in pigs or humans. Due to the novel S-IAV reassortant could be found in pig farms, routine genetic monitoring of S-IAVs is important.

It has been reported that the transmission of human-origin viruses to pigs could contribute the adaptations or mutations for the fitness of the viruses. For example, adaptive mutations of pdmH1N12009 after infection in pigs, the NP gene of the virus develop D53E mutation correlated to less resistance to the antiviral factor in pigs. While some human-origin viruses contain $\mathrm{H} 289 \mathrm{Y}$ substitution to reduce resistance to the antiviral factor and increase viral replication ${ }^{11}$. Some human-origin viruses maintain or loss glycosylation sites in HA protein due to less antibody selection pressure in pigs ${ }^{12}$. Thus genetic analysis of the S-IAVs is important to identify the potential adaptations or mutations that might relate to potential zoonotic of the viruses. The objective of this study was to conduct a longitudinal survey of S-IAVs circulating in a pig farm with history of endemic S-IAV infection from 2017 to 2018. Genetic diversity of S-IAVs and evidence of genetic reassortment of S-IAVs in a pig farm was investigated.

\section{Results}

Survey for swine influenza viruses in a pig farm. In this study, we conducted longitudinal sample collection from a pig farm in central province of Thailand from 2017 to 2018 . This pig farm had a history of endemic S-IAV infection in the past 5 years. Our result showed that $18.81 \%(82 / 436)$ and $19.95 \%(87 / 436)$ of nasal swab samples were positive and suspected for S-IAVs, respectively. By year, S-IAVs could be detected all year round in 2017 which highest in June 2017 (33.33\%). In 2018, S-IAVs could be detected in every month of 2018, except March 2018. Highest percentage of S-IAV positivity was in April 2018 with $40.63 \%$ (13/32). The 169 S-IAV positive and suspected samples were subjected to virus isolation by egg inoculation. Our result showed that 57 influenza viruses $(13.07 \%)$ could be isolated (Supplement Table 1).

Diversity of swine influenza viruses in a pig farm. Twenty four out of 57 swine influenza viruses were selected for whole genome sequencing. The $24 \mathrm{~S}$-IAVs were selected based on time of sample collection, subtypes of the viruses and virus titer (low Ct-value). To identify subtype of S-IAVs, nucleotide sequences of HA and NA genes were compared with nucleotide sequences in the NCBI database by using BLAST program. Our result showed that 24 S-IAVs could be identified as S-IAV-H1N1 $(\mathrm{n}=18)$ and S-IAV-H3N2 $(\mathrm{n}=6)$ (Table 1). Phylogenetic tree of $\mathrm{H} 1$ was constructed by comparing S-IAV-H1N1 $(n=18)$ to 125 references viruses. Phylogenetic tree of $\mathrm{H} 1$ gene showed that all S-IAV-H1N1s in this study were clustered with alpha sublineage (1A.1.2) of the classical swine lineage (Fig. 1A). It is noted that alpha sublineage (1A.1.2) is a common lineage of endemic Thai-S-IAVs. Phylogenetic tree of N1 was constructed by comparing $18 \mathrm{~S}-\mathrm{IAV}-\mathrm{H} 1 \mathrm{~N} 1$ to 70 references viruses. N1 of S-IAV-H1N1s in this study was clustered with either Eurasian avian-like swine lineage $(n=6)$ or pdm09 lineage $(n=12)$ (Fig. 1B). The Eurasian avian-like swine lineage is a common lineage of N1 of endemic S-IAVs in Thailand. Phylogenetic tree of H3 was constructed by comparing S-IAV-H3N2 $(n=6)$ to 142 references viruses. Phylogenetic analysis showed that H3 of S-IAV-H3N2 in this study was clustered with North America lineage, sublineage human-like swine (both $\mathrm{Ha}$ and $\mathrm{Hb}$ ), which is previously identified as common lineages of endemic Thai-S-IAV-H3N2 (Fig. 2A). Phylogenetic tree of N2 was constructed by comparing S-IAV-H3N2 $(n=6)$ to 82 references viruses. The reference of NA2 gene included 2 major lineages; North American lineage and Eurasian swine lineage. The North American lineage contains 2 subclusters: N2-1998 and N2-2002 lineages from human seasonal influenza. Phylogenetic analysis showed that N2 genes of S-IAV-H3N2s were clustered into North American lineage with sublineage human-like swine of endemic Thai S-IAV-H3N2 (Fig. 2B). Phylogenetic trees of the internal genes of the S-IAV-H1N1 and S-IAV-H3N2 were constructed by comparing S-IAV-H1N1 $(\mathrm{n}=18)$ and S-IAV-H3N2 $(n=6)$ to references viruses. The reference viruses included Classical swine lineage, Eurasian swine lineage, North America triple reassortant lineage, human seasonal lineage and pdm09 lineage. Phylogenetic analysis of each internal genes showed that internal genes of S-IAV-H1N1s $(n=18)$ and S-IAV-H3N2s $(n=6)$ were clustered with pdm09 lineage indicating S-IAVs in this study acquired internal genes (backbone) from pdmH1N1-2009 virus (Supplement Figures 1-6).

Gene constellation of the S-IAVs was identified by designated by using the combination of eight lineages or clusters of the virus. Of 24 S-IAVs, we observed 3 genotypes of S-IAVs including (1) rH1N1 (pdm + 1) contained $\mathrm{H} 1$ from classical swine lineage (CS) of endemic Thai-S-IAVs and the other 7 genes (NA and internal genes) from pdm09 lineage. (2) rH1N1 (pdm +2) contained H1 from classical swine lineage (CS) of endemic Thai-S-IAVs, while N1 from Eurasian avian-like swine lineage (EA) of endemic Thai-S-IAVs and the 6 internal genes from pdm09 lineage, and (3) rH3N2 contained HA3 and NA2 genes from human-like swine lineage of endemic ThaiS-IAVs and internal genes from pdm09 lineage (Fig. 3A,B). Our result suggested that internal genes of pdm09 lineage persist and become predominant lineage in endemic Thai-S-IAVs (Figs. 1A and 2A, Supplement Table 2). It should be concerned that persistence of gene especially pdmH1N1-2009 in pigs for long period in the pig farms could contribute the generation of a novel virus with high infectivity and transmissibility in pigs or humans.

For genetic analysis of $\mathrm{H} 1$ cleavage site, S-IAVs in this study had identical H1 cleavage site "PSIQSRGLF" to pdmH1N1-2009 and endemic S-IAVs. For receptor binding sites (HA-190, 225), most S-IAVs contained $190 \mathrm{D}$ and $225 \mathrm{D}$ suggesting preferential binding to human receptor $(2,6 \text { sialic acid receptor })^{13}$. Expect one virus (CU21626) contained E190 suggesting the virus prefer to bind avian receptor $(2,3 \text { sialic acid receptor })^{14}$. Genetic analysis of $\mathrm{H} 1$ antigenic sites ( $\mathrm{Sa}, \mathrm{Sb}, \mathrm{Ca} 1, \mathrm{Ca} 2$ and $\mathrm{Cb}$ ) showed that S-IAVs in this study contain similar amino acids of antigenic sites to endemic Thai-S-IAVs (Table 2). For HA3 genetic analysis, the receptor binding sites (HA3-226 and 228) of S-IAVs in this study and endemic Thai-S-IAVs posed I226 and S228 suggesting preferential binding to human receptor ${ }^{14,15}$. Genetic analysis of antigenic sites of $\mathrm{H} 3$ (A, B, C, D, and E) showed that antigenic sites A (140-146) and B (156-161, 189-199) contained amino acids similar to endemic Thai-S-IAVs (Table 3). For genetic analysis of some internal genes (PB2, M and NS genes), all S-IAVs in this study possessed 


\begin{tabular}{|c|c|c|c|c|c|}
\hline Virus & Subtype $^{\mathrm{a}}$ & Year & Host & Age & GenBank no \\
\hline \multicolumn{6}{|l|}{ S-IAV-H1N1 } \\
\hline A/swine/THA/CU3732/2017 & rH1N1 (pdm + 2) & Jan-17 & Swine & 4 weeks & MT378014-21 \\
\hline A/swine/THA/CU3743/2017 & rH1N1 (pdm +2) & Jan-17 & Swine & 6 weeks & MT378022-29 \\
\hline A/swine/THA/CU3759/2017 & rH1N1 (pdm +2) & Apr-17 & Swine & 4 weeks & MT378030-37 \\
\hline A/swine/THA/CU3764/2017 & rH1N1 (pdm +2) & Apr-17 & Swine & 6 weeks & MT378038-45 \\
\hline A/swine/THA/CU3770/2017 & rH1N1 (pdm +2) & Apr-17 & Swine & 6 weeks & MT378046-53 \\
\hline A/swine/THA/CU3793/2017 & rH1N1 (pdm +2) & Jun-17 & Swine & 4 weeks & MT378062-69 \\
\hline A/swine/THA/CU3802/2017 & rH1N1 (pdm +1) & Jun-17 & Swine & 6 weeks & MT378086-93 \\
\hline A/swine/THA/CU3803/2017 & rH1N1 (pdm +1) & Jun-17 & Swine & 6 weeks & MT378094-01 \\
\hline A/swine/THA/CU3798/2017 & rH1N1 (pdm +1) & Jun-17 & Swine & 4 weeks & MT378078-85 \\
\hline A/swine/THA/CU21299/2018 & rH1N1 $(\mathrm{pdm}+1)$ & Apr-18 & Swine & 6 weeks & MT377918-25 \\
\hline A/swine/THA/CU21304/2018 & rH1N1 (pdm +1) & Apr-18 & Swine & 10 weeks & MT377942-49 \\
\hline A/swine/THA/CU21626/2018 & rH1N1 (pdm +1) & Jun-18 & Swine & 6 weeks & MT377950-57 \\
\hline A/swine/THA/CU21630/2018 & & Jun18 & & 8 weeks & \\
\hline A/swine/THA/CU21970/2018 & rH1N1 (pdm +1) & Jul-18 & Swine & 6 weeks & MT377966-73 \\
\hline A/swine/THA/CU22117/2018 & rH1N1 (pdm +1) & Aug-18 & Swine & 4 weeks & MT377974-81 \\
\hline A/swine/THA/CU22300/2018 & rH1N1 (pdm + 1) & Sep-18 & Swine & 10 weeks & MT377982-89 \\
\hline A/swine/THA/CU22351/2018 & rH1N1 $(\mathrm{pdm}+1)$ & Oct-18 & Swine & 9 weeks & MT377998-05 \\
\hline A/swine/THA/CU22630/2018 & rH1N1 (pdm +1) & Nov-18 & Swine & 4 weeks & MT378006-13 \\
\hline \multicolumn{6}{|l|}{ S-IAV-H3N2 } \\
\hline A/swine/THA/CU3794/2017 & $\mathrm{rH} 3 \mathrm{~N} 2(\mathrm{pdm}+2)$ & Jun-17 & Swine & 4 weeks & MT378102-09 \\
\hline A/swine/THA/CU3816/2017 & rH3N2 (pdm+2) & Jun-17 & Swine & 10 weeks & MT378070-77 \\
\hline A/swine/THA/CU3790/2017 & rH3N2 (pdm+2) & Jun-17 & Swine & 4 weeks & MT378054-61 \\
\hline A/swine/THA/CU20218/2018 & rH3N2 (pdm + 2) & Nov-17 & Swine & 8 weeks & MT377926-33 \\
\hline A/swine/THA/CU20226/2018 & rH3N2 (pdm+2) & Nov-17 & Swine & 12 weeks & MT377934-41 \\
\hline A/swine/THA/CU22337/2018 & rH3N2 (pdm +2) & Oct-18 & Swine & 6 weeks & MT377990-97 \\
\hline
\end{tabular}

Table 1. Description of Thai S-IAV-H1N1 and S-IAV-H3N2 characterized in this study. ${ }^{a} \mathrm{rH} 1 \mathrm{~N} 1(\mathrm{pdm}+1)$ : reassorted S-IAV-H1N1 genotype contained HA gene from classical swine lineage (CS) and other genes from pandemicH1N1-2009 lineage (pdm09). rH1N1 (pdm + 2): reassorted S-IAV-H1N1 genotype contained HA gene from classical swine lineage (CS), NA gene from Eurasian avian-like lineage (EA) and internal genes from pandemicH1N1-2009 lineage (pdm09). rH3N2 (pdm + 2): reassorted S-IAV-H3N2 genotype contained HA and NA genes from human-like swine and internal genes from pandemicH1N1-2009 lineage (pdm09).

glutamic acid (E) at position 627 in PB2 (E627), which correlated with less virulence of the viruses. While all S-IAVs contained aspartic acid (D) at position 701 in PB2 (N701D) and position 92 in NS (E92D), which correlated to the more virulence of the viruses ${ }^{15-17}$. For genetic analysis of antiviral resistance in $\mathrm{M}$ gene at position 31 of $\mathrm{M}$ gene, all S-IAVs in this study possessed amino acid $\mathrm{N}$ at position $31(31 \mathrm{~N})$ suggesting the viral resistance to amantadine ${ }^{18}$. On the other hand, genetic analysis of antiviral resistance (oseltamivir) in NA gene (NA1-119, 275, 293, and 295 and NA2-146, 219, and 272) showed that all S-IAVs in this study are sensitive to oseltamivir (for NA1; E119, H275, R293, N295 and NA2; N146, S219, A272) (data not shown).

For serological test of S-IAVs, the serum samples were tested for antibodies against influenza subtypes H3N2, $\mathrm{H} 1 \mathrm{~N} 1$ and pdmH1N1. Our results showed that serum samples had antibodies against S-IAV-H3N2 (37.23\%; 140/436), S-IAV-H1N1 (18.35\%; 69/436) and pdmH1N1 viruses (27.13\%; 102/436). In detail, the HI titer against S-IAV-H3N2 (endemic Thai-S-IAVs-H3N2) was highest in April 2017 (73.33\%) and in September 2018 (51.52\%). For S-IAV-H1N1, antibody titer against endemic Thai-S-IAV-H1N1 was highest in November $2017(16.67 \%)$ and in August 2018 (29.03\%). For pdmH1N1, HI titer against pH1N1 was highest in November 2017 (50.00\%) and in July 2018 (63.33\%).

\section{Discussion}

In this study, we conducted a longitudinal survey in a pig farm from January 2017 to November 2018. The previous study on S-IAVs in this pig farm in 2015 showed that this pig farm had been infected with S-IAVs and estimated prevalence of S-IAVs was $6.66 \%{ }^{5}$. Comparing to previous study, the S-IAV prevalence in this study was $18.81 \%$ (from January 2017 to November 2018), which was higher than previous report in 2015. The possible explanation is that this study was more focusing on target sample collection in piglets and weaning pigs (4-10 week-old), thus higher prevalence of S-IAVs was observed.

In this study, two subtypes of S-IAVs (S-IAV-H1N1 and S-IAV-H3N2) were identified. Comparing to the previous study in Thailand during 2000-2014, three subtypes of S-IAVs were identified, but S-IAV-H1N2 could not be identified in this study. Notably, S-IAV-H1N2 has lower prevalence than S-IAV-H1N1 and S-IAV-H3N2 ${ }^{5,19}$. Phylogenetic analysis of $\mathrm{H} 1$ gene showed that S-IAVs in this study as well as endemic Thai-S-IAVs belonged to 
$\mathbf{A}$
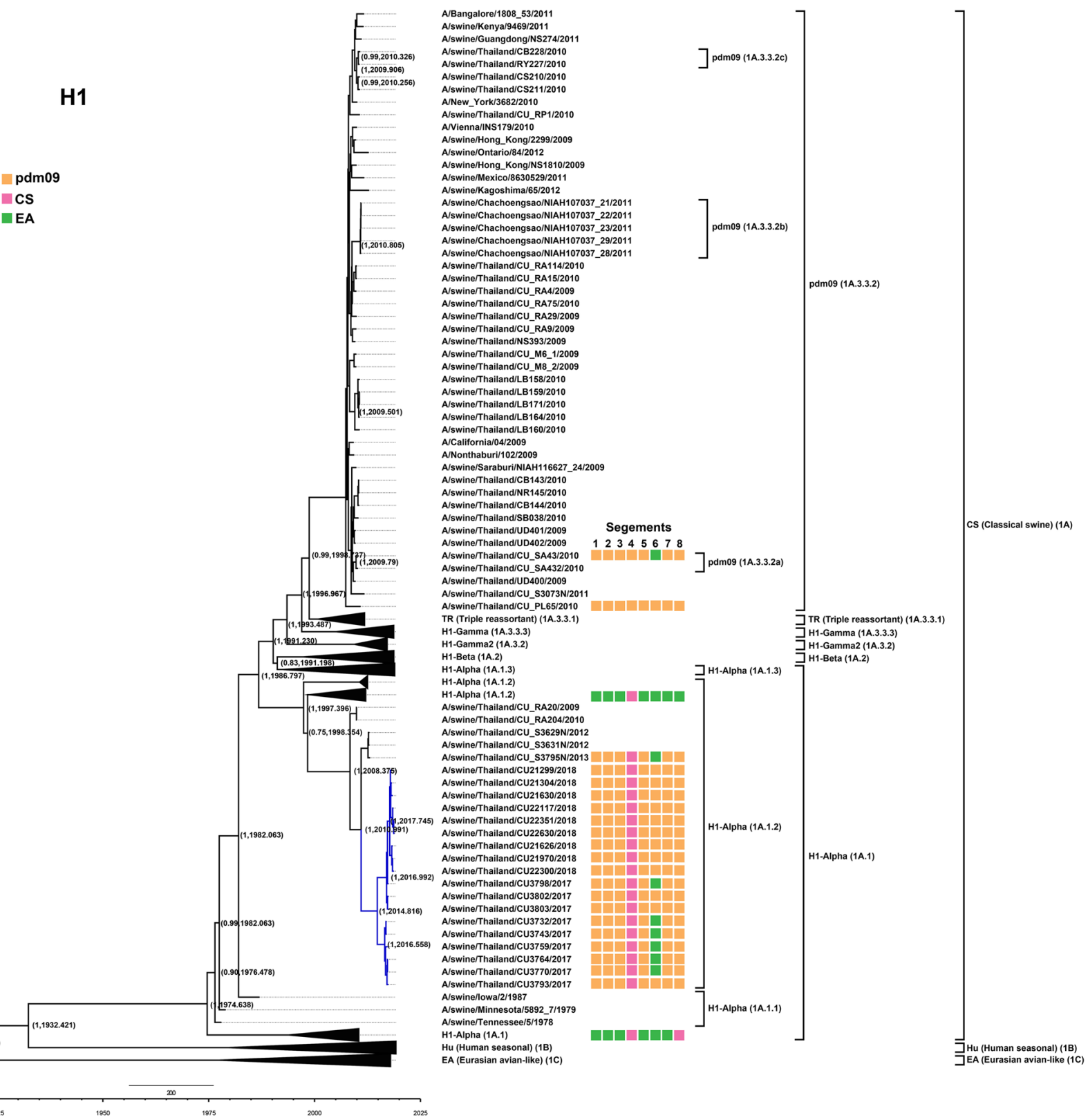

Figure 1. (A) Phylogenetic tree of H1 of S-IAV-H1N1. (B) Phylogenetic tree of N1 of S-IAV-H1N1. Yellow, pink, and green colors indicate pdm2009-H1N1 lineage (pdm09), classical swine lineage (CS), Eurasian avianlike lineage (EA), respectively. Circles indicate S-IAV-H1N1 isolated in this study. The phylogenetic tree was constructed by using the Beast program with Bayesian Markov-Chain Monte Carlo (BMCMC), with 50,000,000 generations and an average standard deviation of split frequencies $<0.10$. Values on branches represent posterior probability and times of most recent common ancestor (TMRCA) among H1-S-IAV. 
B

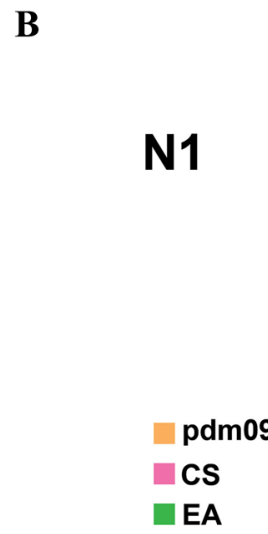

1...

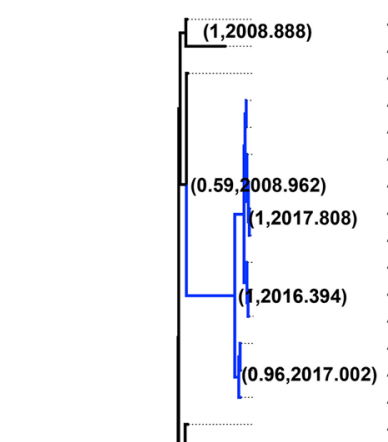

A/California/04/2009

A/Semnan/0781/2015

A/Mexico City/lBT22/2009

A/swine/Thailand/CU21299/2018

A/swine/Thailand/CU21304/2018

A/swine/Thailand/CU21630/2018

A/swine/Thailand/CU22117/2018

A/swine/Thailand/CU22351/2018

A/swine/Thailand/CU22630/2018 A/swine/Thailand/CU21626/2018

A/swine/Thailand/CU21970/2018

A/swine/Thailand/CU22300/2018

A/swine/Thailand/CU3798/2017

A/swine/Thailand/CU3803/2017

A/swine/Thailand/CU3802/2017

A/Nonthaburi/102/2009

A/swine/Thailand/CU_RA114/2010

A/swine/Thailand/CU_RA15/2010

A/swine/Thailand/CU_RA29/2009

A/swine/Thailand/CU_RA9/2009

A/Singapore/KK066/2010

A/swine/Hong_Kong/201/2010

A/swine/Hong_Kong/2299/2009

A/swine/Guangdong/NS274/2011

A/swine/Kenya/9469/2011

A/Vienna/INS179/2010

A/swine/Hong_Kong/NS1810/2009

A/swine/Mexico/8630529/2011

A/swine/Chachoengsao/NIAH107037_21/2011

A/swine/Oklahoma/A01290605/2013

A/swine/Oklahoma/A02214419/2017

A/swine/Guangdong/109/2013

A/swine/Hunan/153/2013

A/swine/Hebei/148/2012

A/swine/Hong_Kong/4999/2011

A/swine/Shanghai/1205/2017

A/swine/Tianjin/42/2011

A/swine/Hong_Kong/1105/2009

A/swine/Hong_Kong/NS29/2009

A/Thailand/271/2005

A/swine/Thailand/CU_SA43/2010

A/swine/Thailand/CU_SA432/2010

A/swine/Chonburi/06CB2/2006

A/swine/Thailand/HF6/2005

A/swine/Chonburi/NIAH9469/2004

A/swine/Chonburi/NIAH977/2004

A/swine/Chachoengsao/NIAH587/2005

A/swine/Ratchaburi/NIAH1481/2000

A/swine/Thailand/CU_S3243N/2012

A/swine/Thailand/CU_S3246N/2012

A/swine/Thailand/CU_S3248N/2012

A/swine/Thailand/CU_S3350N/2012

A/swine/Thailand/CU-RA204/2010

A/swine/Thailand/CU3732/2017

A/swine/Thailand/CU3743/2017

A/swine/Thailand/CU3759/2017

A/swine/Thailand/CU3770/2017

A/swine/Thailand/CU3793/2017

A/swine/Thailand/CU3764/2017

A/swine/Thailand/CU_S3795N/2013

A/swine/Thailand/CU_S3340N/2012

Human seasonal (Hu)

Classical swine (CS)
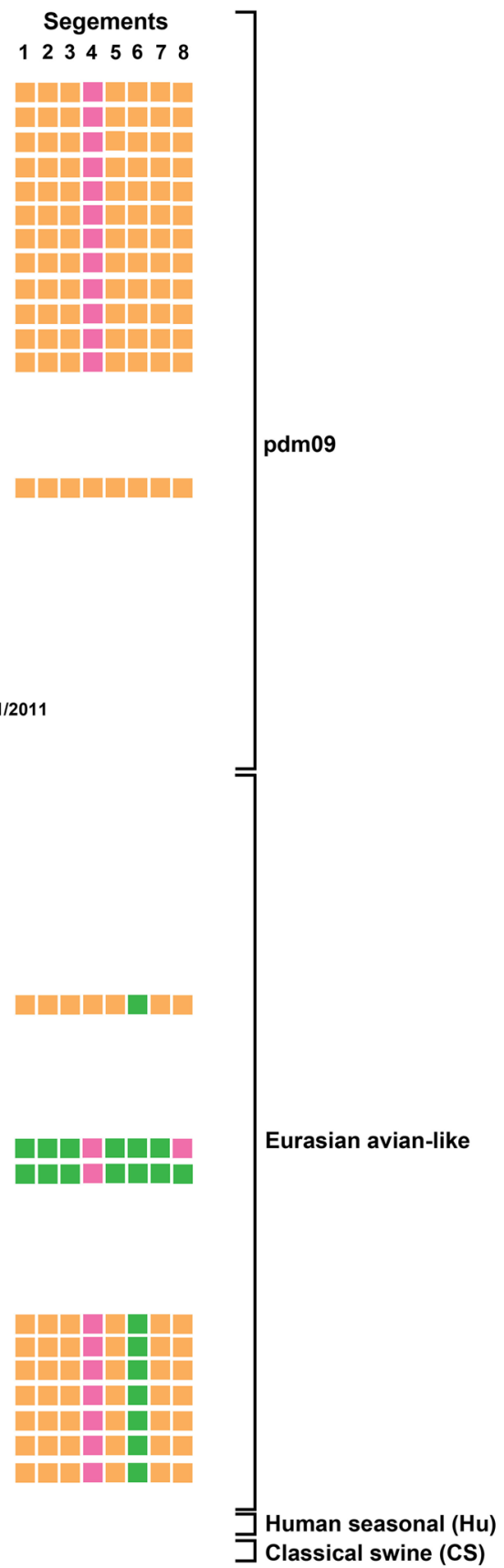

0.0

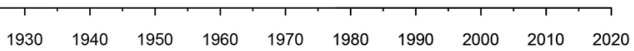

Figure 1. (continued)

the distinct sublineage 1A.1.2 of classical swine $(\mathrm{CS})^{5,9}$. Based on the MCC phylogenetic tree, the H1 gene of S-IAVs in this study was estimated to have separated from endemic S-IAVs-H1N1 since 2010 (Fig. 1A). While $\mathrm{N} 1$ gene of S-IAVs in this study was closely related to either Eurasian avian-like swine lineage (EA) of endemic Thai-S-IAVs or pdm09 lineage of pdmH1N1-2009 virus. This result suggested that pdmH1N1-2009 circulated for a period of time or repeat introduced into this pig farm. Then the pdmH1N1-2009 reassorted with other endemic S-IAVs and contribute N1 gene to novel reassortant influenza viruses. Phylogenetic analysis of $\mathrm{H} 3$ and $\mathrm{N} 2$ genes showed that $\mathrm{H} 3$ and $\mathrm{N} 2$ were clustered with human-like swine viruses that introduced in pigs during 1990s and became the predominant subclusters (Ha and Hb) of endemic S-IAV-H3N2 in Thailand ${ }^{20}$. In this study, HA3 and NA2 genes were still clustered with human-like swine lineage, subclusters $\mathrm{Ha}(\mathrm{n}=1)$ and $\mathrm{Hb}(\mathrm{n}=5)$ suggesting the fitness combination of surface genes ( $\mathrm{H} 3$ and N2) from endemic S-IAVs and internal genes from pdmH1N1-200921. The MCC tree of H3 showed that the S-IAVs in this study was diverged from the endemic S-IAVs-H3N2 since 2012-2013. 

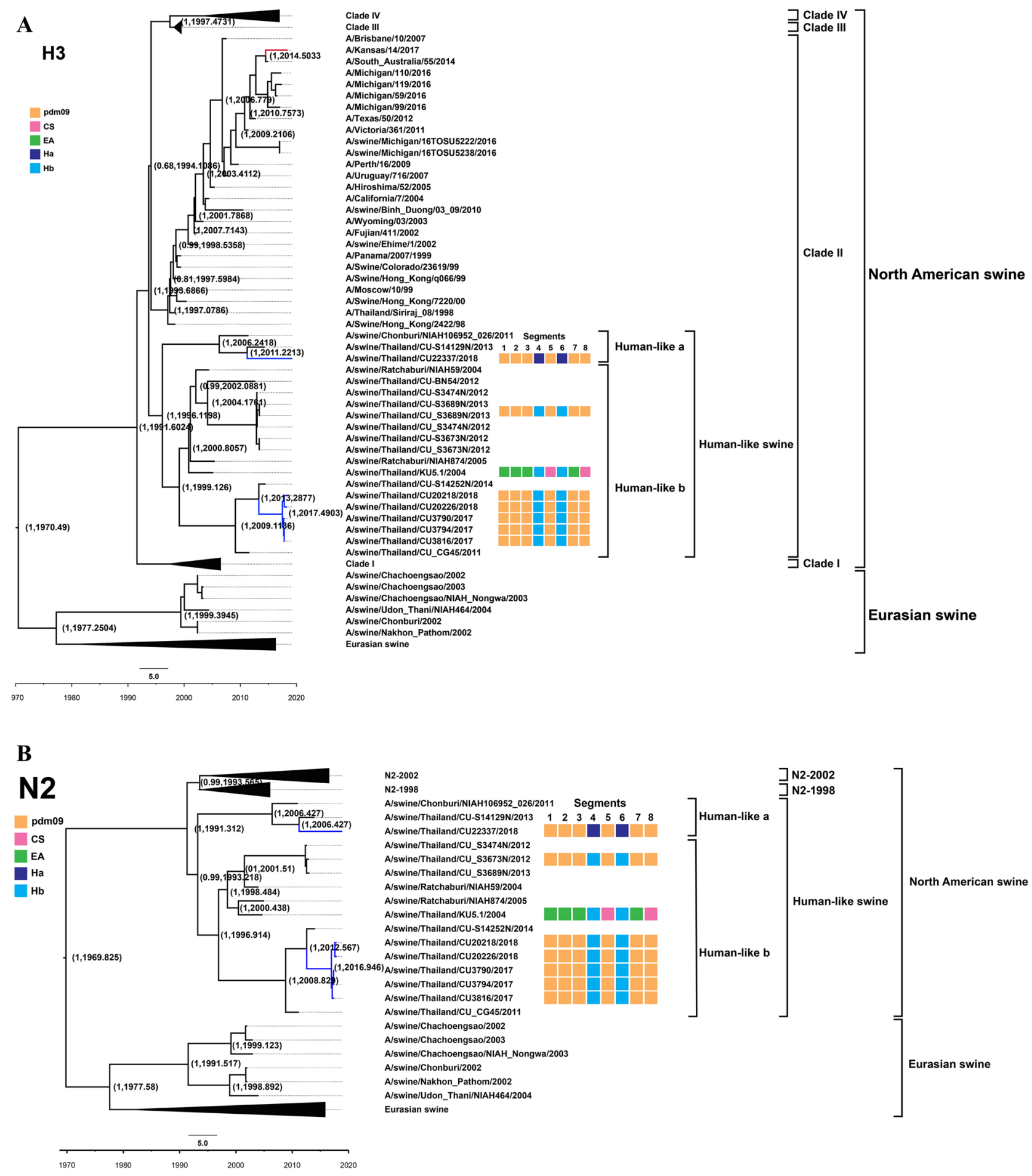

Figure 2. (A) Phylogenetic tree of H3 of S-IAV-H3N2. (B) Phylogenetic tree of N2 of S-IAV-H3N2. Yellow, pink, green, blue and dark blue colors indicate pdm2009-H1N1 lineage (pdm09), classical swine lineage (CS), Eurasian avian-like lineage (EA), Ha human-like swine lineage (Ha), $\mathrm{Hb}$ human-like swine lineage (Hb), respectively. Circles indicate S-IAV-H1N1 isolated in this study. The phylogenetic tree was constructed by using the Beast program with Bayesian Markov-Chain Monte Carlo (BMCMC), with 50,000,000 generations and an average standard deviation of split frequencies $<0.10$. Values on branches represent posterior probability and times of most recent common ancestor (TMRCA) among H3-S-IAV.

In this study, there were 3 reassortant genotypes of S-IAVs; rH1N1 $(\mathrm{pdm}+1), \mathrm{rH} 1 \mathrm{~N} 1(\mathrm{pdm}+2)$ and $\mathrm{rH} 3 \mathrm{~N} 2$ $(\mathrm{pdm}+2)$. In Thailand, at least 7 genotypes have been reported including eH1N1 $(6+2)$, eH1N1 $(7+1), \mathrm{rH} 1 \mathrm{~N} 1$ $(\mathrm{pdm}+1), \mathrm{rH} 1 \mathrm{~N} 1(\mathrm{pdm}+2), \mathrm{rH} 1 \mathrm{~N} 2(\mathrm{pdm}+2), \mathrm{rH} 3 \mathrm{~N} 2(\mathrm{pdm}+2)$ and pdmH1N1 (Supplement Table 2) $)^{5,9,20}$. The genotype $\mathrm{rH} 1 \mathrm{~N} 1(\mathrm{pdm}+2)$ and $\mathrm{rH} 3 \mathrm{~N} 2(\mathrm{pdm}+2)$, which identified in this study, have been previously reported in 
A

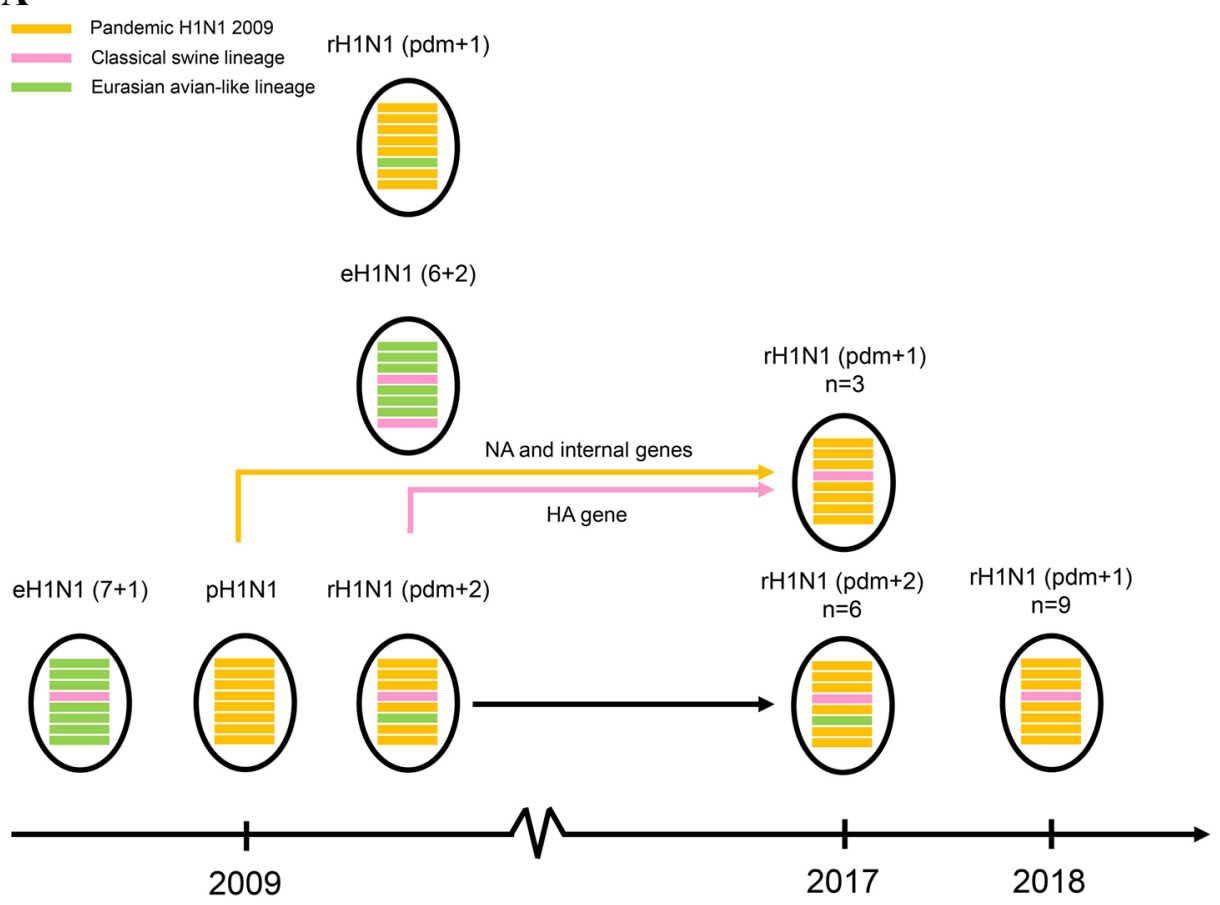

B

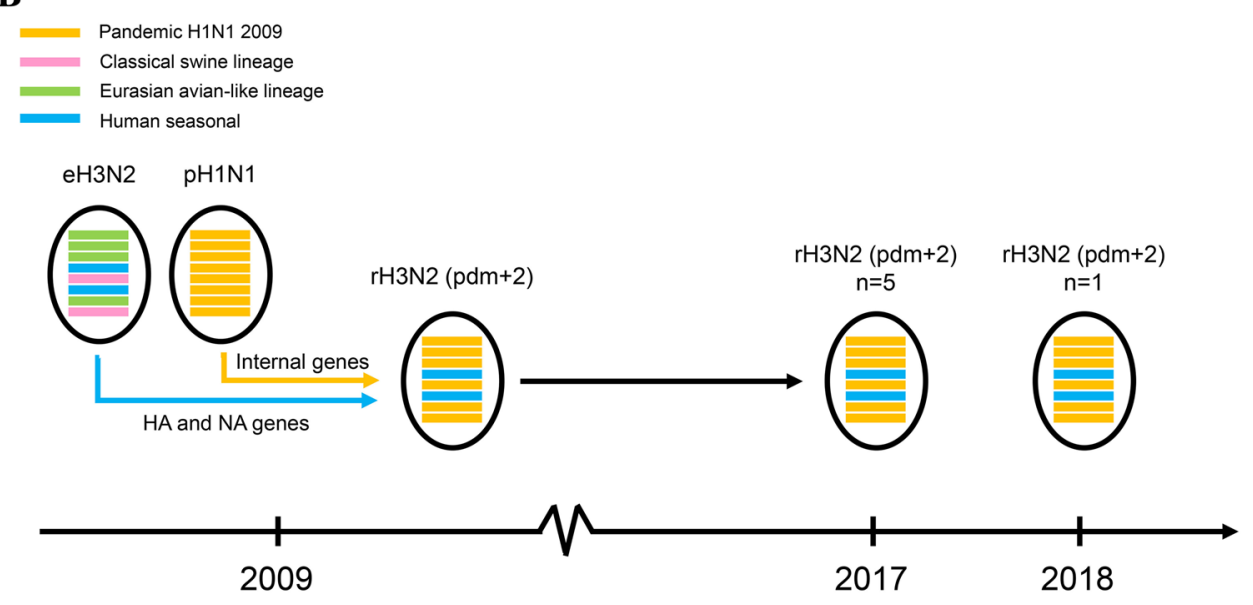

Figure 3. (A) Schematic presentation of genotypes of S-IAV-H1N1 in this study. (B) Schematic presentation of genotypes of S-IAV-H3N2 in this study. Oval represent the virus with 8 gene segments. Lines from top to bottom represent PB2, PB1, PA, HA, NP, NA, M and NS genes. Yellow, pink, green, blue and dark blue colors indicate pdm2009-H1N1 lineage (pdm09), classical swine lineage (CS), Eurasian avian-like lineage (EA), Ha human-like swine lineage $(\mathrm{Ha}), \mathrm{Hb}$ human-like swine lineage $(\mathrm{Hb})$, respectively.

Thailand indicating these genotypes are stable and continuous circulating in a pig farm for several years. In contrast, novel genotype $\mathrm{rH} 1 \mathrm{~N} 1(\mathrm{pdm}+1)$, ( $\mathrm{H} 1$ from classical swine and other 7 genes from pdmH1N1), has never been reported in Thailand. Our result supported the reassortment or multiple-reassortment between endemic Thai-S-IAVs and pdmH1N1-2009. Reassortment of swine influenza viruses have been observed worldwide such as in China ${ }^{22,23}$, Europe ${ }^{24}$ and America ${ }^{25-27}$. Since all genotypes of S-IAVs in this study contain backbone (PB2, PB1, PA, NP, M and NS genes) from pdmH1N1-2009. It could be speculated that the entire pdmH1N1-2009 virus did not persist but the internal genes of pdmH1N1-2009 have became predominant lineage of S-IAVs in Thailand. Thus the predominant of internal genes of pdmH1N1-2009 could stimulate the expand diversity and rapid evolution of S-IAVs in Thailand as well as in the region ${ }^{21}$.

The recent study in China has reported that the predominant reassortant S-IAVs genotype G4 (H1N1 gene from Eurasian Avian-like (HA, NA) and internal genes from pdmH1N1-2009 (PB2, PB1, PA, NP, M) and Triple reassortant internal gene (NS)) showed efficient infectivity and high pathogenicity in experimental animal model. Their findings raise a concern of potential pandemic of the viruses ${ }^{28}$. 


\begin{tabular}{|c|c|c|c|c|c|c|c|c|c|c|c|c|c|c|c|}
\hline \multirow[b]{4}{*}{ Viruses } & \multirow[b]{4}{*}{ Subtype } & \multirow{4}{*}{$\begin{array}{l}\text { H1 } \\
\text { Cluster }\end{array}$} & \multicolumn{13}{|l|}{ H1 gene ${ }^{a}$} \\
\hline & & & \multicolumn{10}{|c|}{ Antigenic site } & \multicolumn{2}{|c|}{$\begin{array}{l}\text { Receptor } \\
\text { binding } \\
\text { site }\end{array}$} & \multirow{3}{*}{$\begin{array}{l}\begin{array}{l}\text { HA } \\
\text { cleavage } \\
\text { site }\end{array} \\
325-333\end{array}$} \\
\hline & & & \multicolumn{3}{|c|}{ Sa } & \multirow{2}{*}{\begin{tabular}{|l|} 
Sb \\
$187-198$
\end{tabular}} & \multicolumn{3}{|l|}{ Cal } & \multicolumn{2}{|l|}{$\mathrm{Ca} 2$} & \multirow{2}{*}{\begin{tabular}{|l|} 
Cb \\
$78-83$ \\
\end{tabular}} & \multirow[b]{2}{*}{190} & \multirow[b]{2}{*}{225} & \\
\hline & & & $128-129$ & $156-160$ & $162-167$ & & 169-173 & 206-208 & 238-240 & $140-145$ & $224-225$ & & & & \\
\hline \multicolumn{16}{|l|}{ Reference } \\
\hline $\begin{array}{l}\text { Califor- } \\
\text { nia/04/2009 }\end{array}$ & pdmH1N1 & $\mathrm{pdm}$ & PN & KKGNS & PKLSKS & $\begin{array}{l}\text { TSADQQS- } \\
\text { LYQNA }\end{array}$ & INDKG & GSS & EPG & PHAGAK & $\mathrm{RD}$ & SLSTAS & $\mathrm{D}$ & $\mathrm{D}$ & $\begin{array}{l}\text { PSIQSR- } \\
\text { GLF }\end{array}$ \\
\hline $\begin{array}{l}\text { THA/ } \\
\text { CUPL65/2010 }\end{array}$ & PdmH1N1 & $\mathrm{pdm}$ & PN & KKGNS & PKLSKS & $\begin{array}{l}\text { TSADQQS- } \\
\text { LYQNA }\end{array}$ & INDKG & GSS & EPG & PHAGAE & $\mathrm{RD}$ & SLSTAS & $\mathrm{D}$ & $\mathrm{D}$ & $\begin{array}{l}\text { PSIQSR- } \\
\text { GLF }\end{array}$ \\
\hline $\begin{array}{l}\text { THA/ } \\
\text { CU3340N/2012 }\end{array}$ & $\begin{array}{l}\text { eH1N1 } \\
(6+2)\end{array}$ & CS & PN & KKGNS & PKLSKS & $\begin{array}{l}\text { TSTDQQS- } \\
\text { LYQNA }\end{array}$ & VNNKK & SSS & EPG & HYAGAN & $\mathrm{RD}$ & LLFKAS & $\mathrm{D}$ & $\mathrm{D}$ & $\begin{array}{l}\text { PSIQSR- } \\
\text { GLF }\end{array}$ \\
\hline $\begin{array}{l}\text { THA/ } \\
\text { NIAH587/2005 }\end{array}$ & $\begin{array}{l}\text { eH1N1 } \\
(7+1)\end{array}$ & CS & PN & KKGNS & PKLSKS & $\begin{array}{l}\text { TNTDQQS- } \\
\text { LYQNA }\end{array}$ & VNNKK & GSS & EPG & PYAGAN & RG & LLFAIN & $\mathrm{D}$ & G & $\begin{array}{l}\text { PSIQSR- } \\
\text { GLF }\end{array}$ \\
\hline $\begin{array}{l}\text { THA/ } \\
\text { CU3795N/2013 }\end{array}$ & $\begin{array}{l}\text { rH1N1 } \\
(6+2)\end{array}$ & CS & PN & KKENS & PKISKS & $\begin{array}{l}\text { TSNDQQS- } \\
\text { LYQNA }\end{array}$ & FNNKG & SSS & KPG & PYAGAN & $\mathrm{RD}$ & LLFNAS & $\mathrm{D}$ & $\mathrm{D}$ & $\begin{array}{l}\text { PSIQSR- } \\
\text { GLF }\end{array}$ \\
\hline $\begin{array}{l}\text { THA/ } \\
\text { CUSA43/2010 }\end{array}$ & $\begin{array}{l}\text { rH1N1 } \\
(7+1)\end{array}$ & $\mathrm{pdm}$ & $\mathrm{PN}$ & KKGNS & PKLSKS & $\begin{array}{l}\text { TSADQQS- } \\
\text { LYQNA }\end{array}$ & INDKG & GSS & EPG & PHAGAK & $\mathrm{RD}$ & SLSTAS & $\mathrm{D}$ & $\mathrm{D}$ & $\begin{array}{l}\text { PSIQSR- } \\
\text { GLF }\end{array}$ \\
\hline $\begin{array}{l}\text { THA/ } \\
\text { CUS3629N/2012 }\end{array}$ & $\begin{array}{l}\text { rH1N1 } \\
(\mathrm{pdm}+2)\end{array}$ & CS & $\mathrm{PN}$ & KKENS & PKISKS & $\begin{array}{l}\text { TSNDQQS- } \\
\text { LYQNA }\end{array}$ & FNNKG & SSS & KPG & PYAGAN & $\mathrm{RD}$ & LLFNAS & $\mathrm{D}$ & $\mathrm{D}$ & $\begin{array}{l}\text { PSIQSR- } \\
\text { GLF }\end{array}$ \\
\hline \multicolumn{16}{|l|}{ This study } \\
\hline $\begin{array}{l}\text { THA/ } \\
\text { CU3759/2017 }\end{array}$ & $\begin{array}{l}\text { rH1N1 } \\
(\mathrm{pdm}+2)\end{array}$ & CS & $\mathrm{PN}$ & KKENS & PKLSKS & $\begin{array}{l}\text { TSNDQQV- } \\
\text { LYQNA }\end{array}$ & FNNKG & SSS & KPE & PYAGAN & $R N$ & SLFNAS & $\mathrm{D}$ & $N$ & $\begin{array}{l}\text { PSIQSR- } \\
\text { GLF }\end{array}$ \\
\hline $\begin{array}{l}\text { THA/ } \\
\text { CU3764/2017 }\end{array}$ & $\begin{array}{l}\text { rH1N1 } \\
(\mathrm{pdm}+2)\end{array}$ & CS & PN & KKENS & PKLSKS & $\begin{array}{l}\text { TSNDQQV- } \\
\text { LYQNA }\end{array}$ & FNNKG & SSS & KPE & PYAGAN & $\mathrm{RD}$ & SLFNAS & $\mathrm{D}$ & $\mathrm{D}$ & $\begin{array}{l}\text { PSIQSR- } \\
\text { GLF }\end{array}$ \\
\hline $\begin{array}{l}\text { THA/ } \\
\text { CU3770/2017 }\end{array}$ & $\begin{array}{l}\text { rH1N1 } \\
(\mathrm{pdm}+2)\end{array}$ & CS & PN & KKENS & PKLSKS & $\begin{array}{l}\text { TSNDQQV- } \\
\text { LYQNA }\end{array}$ & FNNKG & SSS & KPE & PYAGAN & $\mathrm{RD}$ & SLFNAS & $\mathrm{D}$ & $\mathrm{D}$ & $\begin{array}{l}\text { PSIQSR- } \\
\text { GLF }\end{array}$ \\
\hline $\begin{array}{l}\text { THA/ } \\
\text { CU3793/2017 }\end{array}$ & $\begin{array}{l}\text { rH1N1 } \\
(\mathrm{pdm}+2)\end{array}$ & CS & PN & KKENS & PKLSKS & $\begin{array}{l}\text { TSNDQQV- } \\
\text { LYQNA }\end{array}$ & FNNKG & SSS & KPE & PYAGAK & $\mathrm{RD}$ & SLFNAS & $\mathrm{D}$ & $\mathrm{D}$ & $\begin{array}{l}\text { PSIQSR- } \\
\text { GLF }\end{array}$ \\
\hline $\begin{array}{l}\text { THA/ } \\
\text { CU3802/2017 }\end{array}$ & $\begin{array}{l}\text { rH1N1 } \\
(\mathrm{pdm}+1)\end{array}$ & CS & PN & KKENS & PKLSKS & $\begin{array}{l}\text { TSNDQQV- } \\
\text { LYQNA }\end{array}$ & FNNRG & SSS & KPE & PYAGAN & $\mathrm{RD}$ & LLFNAS & $\mathrm{D}$ & $\mathrm{D}$ & $\begin{array}{l}\text { PSIQSR- } \\
\text { GLF }\end{array}$ \\
\hline $\begin{array}{l}\text { THA/ } \\
\text { CU3798/2017 }\end{array}$ & $\begin{array}{l}\text { rH1N1 } \\
(\mathrm{pdm}+1)\end{array}$ & CS & PN & KKENS & PKLSKS & $\begin{array}{l}\text { TSNDQQV- } \\
\text { LYQNA }\end{array}$ & FNNRG & SSS & KPE & PYAGAN & $\mathrm{RD}$ & LLFNAS & $\mathrm{D}$ & $\mathrm{D}$ & $\begin{array}{l}\text { PSIQSR- } \\
\text { GLF }\end{array}$ \\
\hline $\begin{array}{l}\text { THA/ } \\
\text { CU3803/2017 }\end{array}$ & $\begin{array}{l}\text { rH1N1 } \\
(\mathrm{pdm}+1)\end{array}$ & CS & PN & KKENS & PKLSKS & $\begin{array}{l}\text { TSNDQQV- } \\
\text { LYQNA }\end{array}$ & FNNRG & SSS & KPE & PYAGAN & $\mathrm{RD}$ & LLFNAS & $\mathrm{D}$ & $\mathrm{D}$ & $\begin{array}{l}\text { PSIQSR- } \\
\text { GLF }\end{array}$ \\
\hline $\begin{array}{l}\text { THA/ } \\
\text { CU21299/2018 }\end{array}$ & $\begin{array}{l}\text { rH1N1 } \\
(\mathrm{pdm}+1)\end{array}$ & CS & PN & KKENS & PKLSKS & $\begin{array}{l}\text { TSNDQQV- } \\
\text { LYQNA }\end{array}$ & FNNRG & SSS & KPE & PYAGAK & $\mathrm{RD}$ & LLFNAS & $\mathrm{D}$ & $\mathrm{D}$ & $\begin{array}{l}\text { PSIQSR- } \\
\text { GLF }\end{array}$ \\
\hline $\begin{array}{l}\text { THA/ } \\
\text { CU21304/2018 }\end{array}$ & $\begin{array}{l}\text { rH1N1 } \\
(\mathrm{pdm}+1)\end{array}$ & CS & PN & KKENS & PKLSKS & $\begin{array}{l}\text { TSNDQQV- } \\
\text { LYQNA }\end{array}$ & FNNRG & SSS & KPE & PYAGAN & $\mathrm{RD}$ & LLFNAS & $\mathrm{D}$ & $\mathrm{D}$ & $\begin{array}{l}\text { PSIQSR- } \\
\text { GLF }\end{array}$ \\
\hline $\begin{array}{l}\text { THA/ } \\
\text { CU21626/2018 }\end{array}$ & $\begin{array}{l}\text { rH1N1 } \\
(\mathrm{pdm}+1)\end{array}$ & CS & PN & KKENS & PKLSKS & $\begin{array}{l}\text { TSNEQQV- } \\
\text { LYQNA }\end{array}$ & FNNRG & SSS & KPE & PYAGAN & $\mathrm{RD}$ & LLFNAS & $E$ & $\mathrm{D}$ & $\begin{array}{l}\text { PSIQSR- } \\
\text { GLF }\end{array}$ \\
\hline $\begin{array}{l}\text { THA/ } \\
\text { CU21970/2018 }\end{array}$ & $\begin{array}{l}\text { rH1N1 } \\
(\mathrm{pdm}+1)\end{array}$ & CS & $\mathrm{PN}$ & KKENS & PKLSKS & $\begin{array}{l}\text { TSNDQQV- } \\
\text { LYQNA }\end{array}$ & FNNRG & SSS & KPE & PYAGAN & $\mathrm{RD}$ & LLFNAS & $\mathrm{D}$ & $\mathrm{D}$ & $\begin{array}{l}\text { PSIQSR- } \\
\text { GLF }\end{array}$ \\
\hline $\begin{array}{l}\text { THA/ } \\
\text { CU22117/2018 }\end{array}$ & $\begin{array}{l}\mathrm{rH1N1} \\
(\mathrm{pdm}+1)\end{array}$ & CS & PN & KKENS & PKLSKS & $\begin{array}{l}\text { TSNDQQV- } \\
\text { LYQNA }\end{array}$ & FNNRG & SSS & KPE & PYAGAN & $\mathrm{RD}$ & LLFNAS & $\mathrm{D}$ & D & $\begin{array}{l}\text { PSIQSR- } \\
\text { GLF }\end{array}$ \\
\hline $\begin{array}{l}\text { THA/ } \\
\text { CU21630/2018 }\end{array}$ & $\begin{array}{l}\mathrm{rH1N1} \\
(\mathrm{pdm}+1)\end{array}$ & CS & PN & KKENS & PKLSKS & $\begin{array}{l}\text { TSNDQQV- } \\
\text { LYQNA }\end{array}$ & FNNRG & SSS & $\mathrm{KPE}$ & PYAGAN & RG & LLFNAS & D & G & $\begin{array}{l}\text { PSIQSR- } \\
\text { GLF }\end{array}$ \\
\hline $\begin{array}{l}\text { THA/ } \\
\text { CU22300/2018 }\end{array}$ & $\begin{array}{l}\text { rH1N1 } \\
(\mathrm{pdm}+1)\end{array}$ & CS & $\mathrm{PN}$ & KKENS & PKLSKS & $\begin{array}{l}\text { TSNDQQV- } \\
\text { LYQNA }\end{array}$ & FNNRG & SSS & KPE & PYAGAN & $R N$ & LLFNAS & $\mathrm{D}$ & $N$ & $\begin{array}{l}\text { PSIQSR- } \\
\text { GLF }\end{array}$ \\
\hline $\begin{array}{l}\text { THA/ } \\
\text { CU22351/2018 }\end{array}$ & $\begin{array}{l}\text { rH1N1 } \\
(\mathrm{pdm}+1)\end{array}$ & CS & PN & KKENS & PKLSKS & $\begin{array}{l}\text { TSNDQQV- } \\
\text { LYQNA }\end{array}$ & FNNRG & SSS & KPE & PYAGAN & $\mathrm{RD}$ & LLFNAS & $\mathrm{D}$ & $\mathrm{D}$ & $\begin{array}{l}\text { PSIQSR- } \\
\text { GLF }\end{array}$ \\
\hline $\begin{array}{l}\text { THA/ } \\
\text { CU22630/2018 }\end{array}$ & $\begin{array}{l}\text { rH1N1 } \\
(\mathrm{pdm}+1)\end{array}$ & CS & PN & KKENS & PKLSKS & $\begin{array}{l}\text { TSNDQQV- } \\
\text { LYQNA }\end{array}$ & FNNRG & SSS & KPE & PYAGAN & RG & LLFNAS & $\mathrm{D}$ & G & $\begin{array}{l}\text { PSIQSR- } \\
\text { GLF }\end{array}$ \\
\hline $\begin{array}{l}\text { THA/ } \\
\text { CU3732/2017 }\end{array}$ & $\begin{array}{l}\text { rH1N1 } \\
(\mathrm{pdm}+1)\end{array}$ & CS & PN & KKENS & PKLSKS & $\begin{array}{l}\text { TSNDQQV- } \\
\text { LYQNA }\end{array}$ & FNNKG & SSS & KPE & PYAGAN & RG & SLFNAS & $\mathrm{D}$ & $G$ & $\begin{array}{l}\text { PSIQSR- } \\
\text { GLF }\end{array}$ \\
\hline $\begin{array}{l}\text { THA/ } \\
\text { CU3743/2017 }\end{array}$ & $\begin{array}{l}\text { rH1N1 } \\
(\mathrm{pdm}+1)\end{array}$ & CS & PN & KKENS & PKLSKS & $\begin{array}{l}\text { TSNDQQV- } \\
\text { LYQNA }\end{array}$ & FNNKG & SSS & KPE & PYAGAN & $\mathrm{RD}$ & SLFNAS & D & D & $\begin{array}{l}\text { PSIQSR- } \\
\text { GLF }\end{array}$ \\
\hline
\end{tabular}

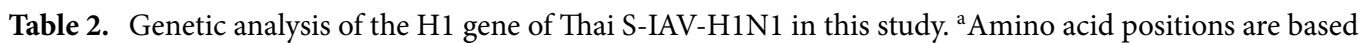
on $\mathrm{H} 3$ numbering.

Based on genetic analysis, the transmission of human-origin viruses to pigs could contribute the adaptations or mutations for the fitness of the viruses. In this study, HA cleavage site, receptor binding sites and antigenic sites of S-IAVs in this study resemble to human viruses, thus the S-IAVs could possibly infect and/or replicate in mammal host including humans. For example, outbreaks of swine origin-H3N2v from pigs to humans and humans to pigs have been documented ${ }^{29}$. In this study, antibodies against H1N1, H3N2 and pdmH1N1 were observed in this pig farm. It is noted that pig had antibody against pdmH1N1, even though the pdmH1N1 viruses 


\begin{tabular}{|c|c|c|c|c|c|c|c|c|c|c|c|}
\hline \multirow[b]{4}{*}{ Viruses } & \multirow[b]{4}{*}{ Subtype } & \multirow[b]{4}{*}{ H3 Cluster } & \multicolumn{9}{|l|}{ H3 gene $^{a}$} \\
\hline & & & \multicolumn{7}{|c|}{ Antigenic site } & \multicolumn{2}{|c|}{$\begin{array}{l}\text { Receptor } \\
\text { binding site }\end{array}$} \\
\hline & & & \multirow{2}{*}{$\begin{array}{l}\text { A } \\
140-146\end{array}$} & \multicolumn{2}{|l|}{ B } & \multirow{2}{*}{$\begin{array}{l}\text { C } \\
277-282\end{array}$} & \multirow{2}{*}{\begin{tabular}{|l|} 
D \\
$205-221$ \\
\end{tabular}} & \multicolumn{2}{|l|}{$\mathbf{E}$} & \multirow[b]{2}{*}{226} & \multirow[b]{2}{*}{228} \\
\hline & & & & $156-161$ & 189-199 & & & 171-175 & $243-249$ & & \\
\hline \multicolumn{12}{|l|}{ Reference } \\
\hline THA/KU5.1/2004 & eH3N2 & $\mathrm{Hu}$ & KRGSVKS & KLDYKY & SDQTNLYVQAS & CNSECI & $\begin{array}{l}\text { STKRSQQTVIP- } \\
\text { NIGSRP }\end{array}$ & NDKFD & LLINSTG & I & S \\
\hline $\begin{array}{l}\text { THA/ } \\
\text { CUS3673N/2012 }\end{array}$ & $\mathrm{rH} 3 \mathrm{~N} 2(\mathrm{pdm}+2)$ & $\mathrm{Hu}$ & KRGSVKS & KLDYKY & NDQTNLYVQAS & CNYGCI & $\begin{array}{l}\text { STKRSQQTVIP- } \\
\text { NIGSRP }\end{array}$ & NDKFN & LLINSTG & I & S \\
\hline $\begin{array}{l}\text { THA/ } \\
\text { CUS14252N/2014 }\end{array}$ & $\mathrm{rH} 3 \mathrm{~N} 2(\mathrm{pdm}+2)$ & $\mathrm{Hu}$ & KRGSVKS & KLDYKY & SDQTNLYVQAS & CNSECI & $\begin{array}{l}\text { STKRSQQTVIP- } \\
\text { NIGFRP }\end{array}$ & NDKFD & LLINSTG & I & S \\
\hline $\begin{array}{l}\text { THA/ } \\
\text { CUS14129N/2013 }\end{array}$ & $\mathrm{rH} 3 \mathrm{~N} 2(\mathrm{pdm}+2)$ & $\mathrm{Hu}$ & KRGYVNS & QSGHKY & SDQTSLYVQAS & CNSECV & $\begin{array}{l}\text { STKRSQQTVIP- } \\
\text { NIGSRP }\end{array}$ & NEKFD & LLINSTG & I & S \\
\hline This study & & $\mathrm{Hu}$ & & & & & & & & & \\
\hline THA/CU3794/2017 & $\mathrm{rH} 3 \mathrm{~N} 2(\mathrm{pdm}+2)$ & $\mathrm{Hu}$ & KRGSVKS & QLNYKY & SDQTNLYVQAS & CNSECI & $\begin{array}{l}\text { STKRSQQTVIP- } \\
\text { NIGFRP }\end{array}$ & NDKFD & LLINSTG & I & S \\
\hline THA/CU3816/2017 & $\mathrm{rH} 3 \mathrm{~N} 2(\mathrm{pdm}+2)$ & $\mathrm{Hu}$ & KRGSVKS & QLNYKY & SDQTNLYVQAS & CNSECI & $\begin{array}{l}\text { STKRSQQTVIP- } \\
\text { NIGFRP }\end{array}$ & NDKFD & LLINSTG & I & S \\
\hline THA/CU3790/2017 & $\mathrm{rH} 3 \mathrm{~N} 2(\mathrm{pdm}+2)$ & $\mathrm{Hu}$ & KRGSVKS & QLNYKY & SDQTNLYVQAS & CNSECI & $\begin{array}{l}\text { STKRSQQTVIP- } \\
\text { NIGFRP }\end{array}$ & NDKFD & LLINSTG & I & S \\
\hline $\begin{array}{l}\text { THA/ } \\
\text { CU20226/2017 }\end{array}$ & $\mathrm{rH} 3 \mathrm{~N} 2(\mathrm{pdm}+2)$ & $\mathrm{Hu}$ & KRGSVKS & QLNYKY & SDQTNLYVQAS & CNSECI & $\begin{array}{l}\text { STKRSQQTVIP- } \\
\text { NIGFRP }\end{array}$ & NDKFD & LLINSTG & I & S \\
\hline $\begin{array}{l}\text { THA/ } \\
\text { CU20218/2017 }\end{array}$ & $\mathrm{rH} 3 \mathrm{~N} 2(\mathrm{pdm}+2)$ & $\mathrm{Hu}$ & KRGSVKS & QLNYKY & SDQTNLYVQAS & CKSECI & $\begin{array}{l}\text { STKRSQQTVIP- } \\
\text { NIGFRP }\end{array}$ & NDKFD & LLINSKG & I & S \\
\hline
\end{tabular}

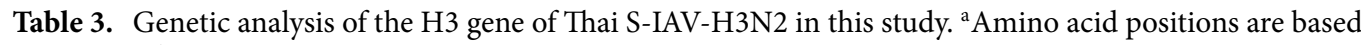
on $\mathrm{H} 3$ numbering.

could not be identified in the pig farm. It has been reported that no cross-reaction between antibodies against $\mathrm{H} 1-\mathrm{S}-\mathrm{IAV}$ and $\mathrm{H} 1$ pandemic viruses ${ }^{30}$. Thus, the HI titer result confirmed that pigs in this farm were exposed to both endemic S-IAV-H1 and S-IAV-H3 and pdmH1N1. It should be noted that the HI antibody titer against pdmH1N1 could be either from pandemic HA already circulating in this swine farm or from pandemic HA that has been recently introduced from human. Based on our findings, we can speculate that reverse zoonotic infection from workers to pigs as well as zoonotic infection from pigs to workers in the pig farm could be occurred. Thus, monitoring of influenza virus in pigs and workers is important and should be routinely conducted. The management in pig farm is important for example personal hygiene, personal protective equipment (PPE), seasonal influenza vaccination should be practiced and used in the pig farms for prevention and control of influenza transmission in pigs and humans.

\section{Materials and methods}

A longitudinal survey of swine influenza viruses in a pig farm. In this study, a longitudinal sample collection was conducted in a pig farm from 2017 to 2018. A pig farm was selected based on a history of S-IAV subtypes $\mathrm{H} 1 \mathrm{~N} 1, \mathrm{H} 1 \mathrm{~N} 2$ and $\mathrm{H} 3 \mathrm{~N} 2$ circulated in a pig farm ${ }^{5}$. The pig farm is located in central province, where considered as high-density pig production area in Thailand. The pig farm is a large-scale pig farm contains approximately 1600 sow and produces 2000 piglets per month with 43 building as farm office, 32 pig housing and 10 worker housing. The farm has open-housing system with moderate biosecurity, which birds and domestic animals can access to pig housing areas. The samples were collected from 436 pigs, including nasal swab samples $(\mathrm{n}=436)$ and blood samples $(\mathrm{n}=436)$ from piglets and nursery pigs $(4-10$-week-old $)$ with clinical signs of S-IAV infection such as coughing, sneezing and nasal discharge. Samples collection was carried out every 4 months in 2017 and every month in 2018 (Supplement Table 1). The nasal swabs were placed in viral transport media (MEM with 7\% BSA, $100 \mathrm{U} / \mathrm{ml}$ penicillin, $100 \mathrm{mg} / \mathrm{ml}$ streptomycin and $1 \mathrm{mg} / \mathrm{ml}$ trypsin). The blood samples were withdraw from jugular vein and placed in $5 \mathrm{ml}$ tubes. The samples were kept on ice and transported to the laboratory within $24 \mathrm{~h}$. The Chulalongkorn University, Animal Care and Uses Protocol committee approved the animal study (CU-VET IACUC \#1831103). All animal study procedures were performed in accordance with CU-VET IACUC guidelines and regulations.

Detection and isolation of swine influenza virus. Viral RNA was extracted from nasal swab samples $(\mathrm{n}=436)$ by using GeneAll GENTi Viral DNA/RNA Extraction Kit (GeneAll; Lisbon, Portugal) on a Genti (GeneAll; Lisbon, Portugal). For influenza A virus detection, one-step Real-time RT-PCR was performed by using SuperScript III Platinum One-Step Quantitative RT-PCR System (Invitrogen; California, USA). The one-step Real-time RT-PCR protocol with M gene specific primers and probes was carried out as previously descripted $^{31}$. Real-time RT-PCR result was interpreted by cycle threshold (Ct- value) of $<36$ as positive, Ct-value of 36-40 as suspected, and Ct-value of $>40$ as negative.

For S-IAV isolation, the real-time RT-PCR positive $(n=82)$ and suspected $(n=51)$ samples were subjected to virus isolation by egg inoculation following WHO recommendation ${ }^{32}$. For egg inoculation, each 100 ul of nasal 
swab sample was inoculated into 3 embryonated chicken eggs (9 to 11-day-old). After $72 \mathrm{~h}$ incubation at $37^{\circ} \mathrm{C}$, allantoic fluid was collected and tested for hemagglutinating activity by HA test. For HA test interpretation, sample with $\mathrm{HA}$ titer of $\geq 4 \mathrm{HA}$ unit per $50 \mu \mathrm{l}$ was interpret positive. For virus confirmation, RNA samples were tested for influenza virus by using one-step Real-time RT-PCR.

Characterization of swine influenza virus. For genetic characterization of S-IAVs, 24 IAVs were subjected to whole genome sequencing by next-generation sequencing (NGS). The criteria of selection of 24 S-IAVs for characterization were based on time of sample collection, subtypes of S-IAVs and high virus titer (low Ctvalue). To perform whole genome sequencing, eight segments of S-IAVs were amplified by using one-step RTPCR with SuperScript III RT-PCR system with Plantinum Taq DNA polymerase (Invitrogen; California, USA) with MBT12 and MBT13 primers $^{33}$. Purified PCR products were quantified and submitted to Novogene co. LTD for Illumina Hiseq PE150 (Illumina Corporation, San Diego, California, USA) using NEBNext Multiplex Oligos for Illumina (New England BioLabs, Ipswich, Massachusetts, USA). To validate and determine nucleotide sequences of eight gene segments of influenza A virus, each nucleotide sequence read, $150 \mathrm{bp}$ each (read) was trimmed to remove adaptors. Then nucleotide sequences were assembly using de-novo assembly method by CLC genomics workbench software Version 11.0.1. (QIAGEN; Hilden, Germany). The sequence contigs were compared with sequence database by BLAST. After the references influenza viruses were selected from BLAST results, the trimmed sequences were used for read mapping to references. Finally, the whole genome sequences were extracted to FASTA format (.fas) by CLC genomics workbench software. Nucleotide sequences of 8 gene segments of swine influenza virus were submitted to the GenBank database under accession number \# MT377918-MT378109 (Table 1).

For phylogenetic analysis, the nucleotide sequences of each gene of S-IAVs from this study were compared with reference S-IAVs. The reference nucleotide sequences of S-IAVs were obtained from Influenza Research Database (https://www.fludb.org). The reference S-IAVs were selected to represent clades/clusters, geographic locations and times of isolation of the viruses. For $\mathrm{H} 1$ gene, the reference viruses include the S-IAVs of Classical swine lineage, CS (1A), human seasonal lineage, Hu (1B), Eurasian avian-like swine lineage, EA (1C). The Classical swine lineage (1A) can be further divided into 6 sub-clusters: alpha (1A.1), beta (1A.2), gamma1 (1A.3.3.3), gamma2 (1A.3.2), pdm09 (1A.3.3.2) and North American triple reassortant (TR) lineage lineages ${ }^{3}$. For HA3 gene, the reference viruses include the S-IAVs of North America lineage and Eurasian swine lineage. The North American lineage contains 4 clades of S-IAVs (Clade I, II, III, IV). The Clade IV of North American S-IAV can be further divided into 6 sub-clusters: A-F. For NA1 gene, the reference viruses include the S-IAVs of Classical swine lineage (CS), human seasonal lineage (Human seasonal), North American triple reassortant (TR) lineage and Eurasian avian-like swine lineage (EA). For NA2 gene, the reference viruses include the S-IAVs of North American and Eurasian swine lineages. The North American lineage can be further divided into 2 sub-groups based on year: 1998 from earlier H3N2 introduction (N2-1998-2012) and 2002 from 2000s human seasonal H1 introduction (N2-2012-2016). For internal genes, the reference viruses include the S-IAVs of Classical swine lineage, human seasonal lineage, North American triple reassortant and Eurasian swine lineage.

To construct phylogenetic trees, the nucleotide sequences of each gene of the viruses (both references viruses and viruses form this study) were aligned with Muscle program v3.6 ${ }^{34}$ in MEGA v7.0 software ${ }^{35}$. Phylogenetic analysis of HA and NA gene of S-IAV was performed by using a BEAST 2.0 program applying a Bayesian Markov Chain Monte Carlo (BMCMC) algorithm. The best-fit substitution model was implemented by bModelTest (Bayesian model test package for BEAST 2). A strict clock model with coalescent constant population was used as model parameters. The Bayesian MCMC chain lengths were 50,000,000 generations, with sampling every 10,000 generations. The tree iteration was discharged with $10 \%$ of the chains as a burn-in pattern by using a tree annotator. The resulting MCC tree was drawn with FigTree software (v1.4.2) (Molecular evolution, phylogenetic and epidemiology, Edinburgh, Scotland, UK). Posterior probability and times of most recent common ancestor (TMRCA) among S-IAVs are provided on branches of trees. Phylogenetic analysis of each internal gene of S-IAV was performed by using MEGA v.7.0 (Tempe, AZ, USA) with neighbor-joining method with Kimura 2-parameter with 1000 bootstrap replicates and Beast program with Bayesian Markov chain Monte Carlo (BMCMC) with $50,000,000$ generations and an average standard deviation of split frequencies $<0.05$. Substitution rates among sites were set in uniform rate and gabs in the sequences were treated in pairwise deletion. To assign genotype of the S-IAVs, lineages or clusters of each gene of the virus were assigned based on the comparison to reference viruses. After lineages or clusters of gene are assigned, the combination of eight lineages or clusters was assigned as genotype or genetic constellation of S-IAVs. The genotypes of the S-IAVs can help to identify reassortment and genetic diversity of the viruses.

Serological test for antibodies against swine influenza virus. Hemagglutination inhibitor test (HI test) was used for detecting antibodies against S-IAVs. In this study, HI test was performed to detect antibodies against 3 reference antigens including endemic S-IAV-H1N1 (A/swine/Thailand/CU-CB1/06), pandemic H1N12009 (A/swine/Thailand/CU-RA29/2009) and endemic S-IAV-H3N2 (A/swine/Thailand/CU-CB8.4/2007). In detail, the serum sample was incubated at $56^{\circ} \mathrm{C}$ for $30 \mathrm{~min}$ to remove heat-labile non-specific factor. Then $100 \mu \mathrm{l}$ of serum sample was treated to remove non-specific inhibitor. For HI test of S-IAV-H3N2 virus, receptor destroying enzyme (RDE) was used to treat serum (50 $\mu$ l of serum: $150 \mu \mathrm{l}$ of RDE) and incubated at $37^{\circ} \mathrm{C}$ for $24 \mathrm{~h}$ and inactivated at $56^{\circ} \mathrm{C}$ for $1 \mathrm{~h}$. The RDE-treated serum was mixed with $100 \mu \mathrm{l}$ of $50 \%$ chicken erythrocyte and incubated at room temperature for $1 \mathrm{~h}$. After centrifuging at $2000 \mathrm{rpm}$ for $10 \mathrm{~min}$, the supernatant was free from non-specific inhibitor serum. For HI test of S-IAV-H1N1 and pdmH1N1-2009, 20\% of kaolin was used to treat serum ( $100 \mu \mathrm{l}$ of serum: $400 \mu \mathrm{l}$ of $20 \%$ kaolin) and incubated at $25^{\circ} \mathrm{C}$ for $30 \mathrm{~min}$. For sedimentation of kaolin, the kaolin-treated serum was centrifuged at $2000 \mathrm{rpm}$ for $10 \mathrm{~min}$. The serum was added with $100 \mu \mathrm{l}$ of $50 \%$ 
chicken erythrocyte and incubated at $25^{\circ} \mathrm{C}$ for $1 \mathrm{~h}$. After centrifuging at $2000 \mathrm{rpm}$ for $10 \mathrm{~min}$, the supernatant was free from non-specific inhibitor serum. To perform HI test, reference antigens were prepared to 8 HA unit per $50 \mu \mathrm{l}$. First $50 \mu \mathrm{l}$ of treated serum was mixed with $50 \mu \mathrm{l}$ of PBS in the first well and serially twofold diluted. $50 \mu \mathrm{l}$ of reference serum was added in each well and incubated at $25^{\circ} \mathrm{C}$ for $45 \mathrm{~min} .100 \mu \mathrm{l}$ of $0.5 \%$ chicken erythrocyte was added into each well. After incubating at $25^{\circ} \mathrm{C}$ for $1 \mathrm{~h}$, the samples with $\mathrm{HI}$ titer of $\geq 1: 40$ were interpreted as positive against specific S-IAVs subtypes.

Ethics statement. This study was conducted under the approval of the Institute for Animal Care and Use Protocol of the Chulalongkorn University (CU-VET; IACUC\#1831103). All animal study procedures were performed in accordance with CU-VET IACUC guidelines and regulations.

\section{Data availability}

The nucleotide sequence data that support the findings of this study are openly available in the GenBank database at https://www.ncbi.nlm.nih.gov/genbank/, accession numbers \# MT377918-MT378109.

Received: 9 May 2020; Accepted: 29 October 2020

Published online: 16 November 2020

\section{References}

1. Schultz-Cherry, S., Olsen, C. W. \& Easterday, B. C. History of swine influenza. Curr. Top. Microbiol. Immunol. 370, 21-28. https:// doi.org/10.1007/82_2011_197(2013).

2. Chutinimitkul, S. et al. Genetic characterization of H1N1, H1N2 and H3N2 swine influenza virus in Thailand. Arch. Virol. https ://doi.org/10.1007/s00705-008-0097-7 (2008).

3. Anderson, T. K. et al. A phylogeny-based global nomenclature system and automated annotation tool for H1 hemagglutinin genes from swine influenza A viruses. mSphere 1, e00275-e216. https://doi.org/10.1128/mSphere.00275-16 (2016).

4. Kitikoon, P. et al. Brief report: Molecular characterization of a novel reassorted pandemic H1N1 2009 in Thai pigs. Virus Genes 43, 1-5. https://doi.org/10.1007/s11262-011-0597-5 (2011).

5. Nonthabenjawan, N. et al. Genetic diversity of swine influenza viruses in Thai swine farms, 2011-2014. Virus Genes 50, 221-230. https://doi.org/10.1007/s11262-014-1153-x (2015).

6. Vincent, A. et al. Review of influenza A virus in swine worldwide: A call for increased surveillance and research. Zoonoses Public Health 61, 4-17. https://doi.org/10.1111/zph.12049 (2014).

7. Howden, K. J. et al. An investigation into human pandemic influenza virus (H1N1) 2009 on an Alberta swine farm. Can. Vet. J. 50, 1153-1161 (2009).

8. Weingartl, H. M. et al. Genetic and pathobiologic characterization of pandemic H1N1 2009 influenza viruses from a naturally infected swine herd. J. Virol. 84, 2245-2256 (2010).

9. Charoenvisal, N. et al. Genetic characterization of Thai swine influenza viruses after the introduction of pandemic H1N1 2009. Virus Genes 47, 75-85 (2013).

10. Hiromoto, Y. et al. Isolation of the pandemic (H1N1) 2009 virus and its reassortant with an H3N2 swine influenza virus from healthy weaning pigs in Thailand in 2011. Virus Res. 169, 175-181. https://doi.org/10.1016/j.virusres.2012.07.025 (2012).

11. Manz, B. et al. Pandemic influenza A viruses escape from restriction by human MxA through adaptive mutations in the nucleoprotein. PLoS Pathog. 9, e1003279. https://doi.org/10.1371/journal.ppat.1003279 (2013).

12. Abe, Y. et al. Effect of the addition of oligosaccharides on the biological activities and antigenicity of influenza A/H3N2 virus hemagglutinin. J. Virol. 78, 9605-9611. https://doi.org/10.1128/JVI.78.18.9605-9611.2004 (2004).

13. Connor, R. J., Kawaoka, Y., Webster, R. G. \& Paulson, J. C. Receptor specificity in human, avian, and equine $\mathrm{H} 2$ and $\mathrm{H} 3$ influenza virus isolates. Virology 205, 17-23. https://doi.org/10.1006/viro.1994.1615 (1994).

14. Vines, A. et al. The role of influenza A virus hemagglutinin residues 226 and 228 in receptor specificity and host range restriction. J. Virol. 72, 7626-7631 (1998).

15. Gao, Y. et al. Identification of amino acids in HA and PB2 critical for the transmission of $\mathrm{H} 5 \mathrm{~N} 1$ avian influenza viruses in a mammalian host. PLoS Pathog. 5, e1000709-e1000709. https://doi.org/10.1371/journal.ppat.1000709 (2009).

16. Hatta, M., Gao, P., Halfmann, P. \& Kawaoka, Y. Molecular basis for high virulence of Hong Kong H5N1 influenza A viruses. Science 293, 1840. https://doi.org/10.1126/science.1062882 (2001).

17. Steel, J., Lowen, A. C., Mubareka, S. \& Palese, P. Transmission of influenza virus in a mammalian host is increased by PB2 amino acids 627K or 627E/701N. PLoS Pathog. 5, e1000252-e1000252. https://doi.org/10.1371/journal.ppat.1000252 (2009).

18. Saito, R. et al. Frequency of amantadine-resistant influenza A viruses during two seasons featuring cocirculation of H1N1 and H3N2. J. Clin. Microbiol. 41, 2164-2165. https://doi.org/10.1128/jcm.41.5.2164-2165.2003 (2003).

19. Choi, Y. K., Goyal, S. M. \& Joo, H. S. Prevalence of swine influenza virus subtypes on swine farms in the United States. Adv. Virol. 147, 1209-1220. https://doi.org/10.1007/s00705-002-0788-4 (2002).

20. Takemae, N. et al. Genetic diversity of swine influenza viruses isolated from pigs during 2000 to 2005 in Thailand. Influenza Other Respir. Viruses 2, 181-189. https://doi.org/10.1111/j.1750-2659.2008.00062.x (2008).

21. Liang, H. et al. Expansion of genotypic diversity and establishment of $2009 \mathrm{H} 1 \mathrm{~N} 1$ pandemic-origin internal genes in pigs in China. J. Virol. 88, 10864-10874. https://doi.org/10.1128/JVI.01327-14 (2014).

22. Cai, M. et al. Molecular evolution of H1N1 swine influenza in Guangdong, China, 2016-2017. Infect. Genet. Evol. 60, 103-108. https://doi.org/10.1016/j.meegid.2018.02.029 (2018).

23. Lam, T.T.-Y. et al. Reassortment Events among swine influenza A viruses in China: Implications for the origin of the 2009 influenza pandemic. J. Virol. 85, 10279. https://doi.org/10.1128/JVI.05262-11 (2011).

24. Zell, R., Scholtissek, C. \& Ludwig, S. In Swine Influenza (eds Richt. J.A. \& Webby, R.J.) 29-55 (Springer, Berlin, 2013).

25. Ducatez, M. F. et al. Multiple reassortment between pandemic (H1N1) 2009 and endemic influenza viruses in pigs, United States. Emerg. Infect. Dis. 17, 1624-1629. https://doi.org/10.3201/eid1709.110338 (2011).

26. Nelson, M. I. et al. Human-origin influenza A(H3N2) reassortant viruses in swine, Southeast Mexico. Emerg. Infect. Dis. 25, 691-700. https://doi.org/10.3201/eid2504.180779 (2019).

27. Rajão, D. S. et al. Reassortment between swine H3N2 and 2009 pandemic H1N1 in the United States resulted in influenza A viruses with diverse genetic constellations with variable virulence in pigs. J. Virol. 91, e01763-e11716. https://doi.org/10.1128/JVI.01763 -16 (2017).

28. Sun, H. et al. Prevalent Eurasian avian-like H1N1 swine influenza virus with 2009 pandemic viral genes facilitating human infection. Proc. Natl. Acad. Sci. USA https://doi.org/10.1073/pnas.1921186117 (2020).

29. Jhung, M. A. et al. Outbreak of variant influenza A(H3N2) virus in the United States. Clin. Infect. Dis. 57, 1703-1712. https://doi. org/10.1093/cid/cit649 (2013). 
30. Sreta, D. et al. Retrospective swine influenza serological surveillance in the four highest pig density provinces of Thailand before the introduction of the 2009 pandemic Influenza A virus subtype H1N1 using various antibody detection assays. J. Vet. Diagn. Investig. 25, 45-53. https://doi.org/10.1177/1040638712466554 (2012).

31. Spackman, E. et al. Development of a real-time reverse transcriptase PCR assay for type A influenza virus and the avian H5 and H7 hemagglutinin subtypes. J Clin Microbiol 40, 3256-3260 (2002).

32. WHO. (World Health Organization, Geneva, 2002).

33. Zhou, B. et al. Single-reaction genomic amplification accelerates sequencing and vaccine production for classical and swine origin human influenza A viruses. J. Virol. 83, 10309-10313. https://doi.org/10.1128/jvi.01109-09 (2009).

34. Edgar, R. C. MUSCLE: Multiple sequence alignment with high accuracy and high throughput. Nucleic Acids Res. 32, $1792-1797$. https://doi.org/10.1093/nar/gkh340 (2004).

35. Kumar, S., Stecher, G. \& Tamura, K. MEGA7: Molecular evolutionary genetics analysis version 7.0 for bigger datasets. Mol. Biol. Evol. 33, 1870-1874. https://doi.org/10.1093/molbev/msw054 (2016).

\section{Acknowledgements}

We would like to thank Chulalongkorn University for supporting the 90th year anniversary scholarship for the first author $(\mathrm{CN})$. Chulalongkorn University supported the One Health Research Cluster and the Center of Excellence for Emerging and Re-emerging Infectious Diseases in Animals (CUEIDAs). The Thailand Research Fund (TRF) supported grant for senior scholar to corresponding author (Prof. Dr. Alongkorn Amonsin) (RTA6080012).

\section{Author contributions}

C.N., S.B., and N.B. performed sample collection, molecular detection, characterization and analysis. T.J. and S.C. participated in whole genome sequencing and phylogenetic analysis. D.P. and A.T. performed serological test and analysis. C.N. and A.T. drafted the manuscript. A.A. (PI) designed the study, performed data analysis, drafted, revised and approved the manuscript. All authors reviewed the manuscript.

\section{Competing interests}

The authors declare no competing interests.

\section{Additional information}

Supplementary information is available for this paper at https://doi.org/10.1038/s41598-020-76771-2.

Correspondence and requests for materials should be addressed to A.A.

Reprints and permissions information is available at www.nature.com/reprints.

Publisher's note Springer Nature remains neutral with regard to jurisdictional claims in published maps and institutional affiliations.

(c) (i) Open Access This article is licensed under a Creative Commons Attribution 4.0 International License, which permits use, sharing, adaptation, distribution and reproduction in any medium or format, as long as you give appropriate credit to the original author(s) and the source, provide a link to the Creative Commons licence, and indicate if changes were made. The images or other third party material in this article are included in the article's Creative Commons licence, unless indicated otherwise in a credit line to the material. If material is not included in the article's Creative Commons licence and your intended use is not permitted by statutory regulation or exceeds the permitted use, you will need to obtain permission directly from the copyright holder. To view a copy of this licence, visit http://creativecommons.org/licenses/by/4.0/.

(C) The Author(s) 2020 